\title{
Marion Eggert
}

\section{Schwalbenflug in Gedichten von Li Bai und Chasper Po}

\section{The Flight of the Swallows in Poems by Li Bai and Chasper Po}

The swallow is said to symbolise domestic bliss, but even more bliss lies in witnessing its flight, which resembles the flight of the imagination - a sudden flash of presence. Here, the flying swallow is akin to the workings of the poetic mind and, more specifically, to metaphor, which 'carries home' meanings that are otherwise unattainable. This essay looks at the swallow metaphor in one poem each by two poets of widely diverging times and cultures: the eminent Li Bai of eighth-century China, and the more recent Romansh poet Chasper Po. We demonstrate how their poems share a deep structural similarity that is founded both in the phenomenological characteristics of their object of observation, the swallow, and in their own, albeit temporary, identification with this bird that builds homes just with its own 'mouth'.

Schwalben fliegen durch die Gedichte von Li Bai und von Chasper Po - nicht in überwältigender Zahl, aber in wahrnehmbaren Bögen. Es ist ihr Nestbau, der die Schwalben als Metapher in die Dichtung trägt, und ihre treue Rückkehr an den heimatlichen Ort - die aber den Aufbruch und die weite Reise voraussetzt. So verbindet sich in ihnen das Nahe mit dem Fernen. Welche Verbindungen schaffen die überraschenden Linien ihres wendigen Flugs zwischen Dichtern, die sonst nur durch den lautlichen Anklang ihrer Namen verbunden scheinen?

In Ost und West scheint die Schwalbe in erster Linie mit ihrem Nestbau und mit ihrer Ortstreue assoziiert zu werden - sie sei ein gutes Omen, heißt es, das für Kindersegen, Wohlstand und häusliches Glück stehe. ${ }^{1}$ Das Glück, das die Schwalbe mit sich bringt, ist jedoch zunächst einmal die Euphorie, die die rauschhafte Wendigkeit ihres Fluges beim Betrachter auslöst. Überwältigender als ihre baumeisterlichen Fähigkeiten sind ihre Flugkünste; als treue Heimkehrerin kann sie uns nur beeindrucken, weil sie fortzieht. Und so wie in das Glück der Zeugenschaft ihres vollkommenen Fluges auch Melancholie gemischt ist - weil er unerreichbar ist, und nicht von

\section{DOI: $10.26045 /$ po-006}

1 So liest man es in gängigen Referenzwerken für Deutschland und Europa; ganz ähnlich klingt, was Eberhard in seinem Lexikon chinesischer Symbolik für China notiert. Wolfram Eberhard: Lexikon chinesischer Symbole. Geheime Sinnbilder in Kunst und Literatur, Leben und Denken der Chinesen, Köln: Diederichs, 1983, S. 260 f. 
Dauer -, so ist auch die Freude an ihrer Wiederkehr immer von dem Wissen um ihren Abschied durchzogen. So erscheint die Schwalbe als ein Wesen des Dazwischen, zwischen Himmel und Erde, zwischen Heimat und Fremde, zwischen dem Vertrauten und dem Überraschenden.

Die Schwalbe ist das Sinnbild der Leichtigkeit - »Fliegende Schwalbe« ([Zhao] Feiyan 趙飛燕, ca. 41-3 v. Chr.) hieß jenes Singmädchen der chinesischen Antike, das bis heute für die überschlanke Schönheit steht -, aber ins Bild wird sie gesetzt mit dem Schweren als Gegenpol. Dies sagen uns zumindest zwei ostasiatische Tuscheskizzen, von Utagawa Hiroshige (安藤廣重1797-1858) und Qi Baishi (齊白石 1864-1957). Hiroshige zeigt in der rechten Hälfte eines querformatigen Bildes vor einem monochromen, gen Boden ins Nichts verlaufenden Fichtenstamm zwei Frauengestalten in überdimensionierter Regenschutzkleidung und schweren Hüten, in einander zugewandter Bewegung. Die linke Bildhälfte ist nahezu leer - bis auf eine angedeutete Wolke und vor ihr, weit oben im äußersten Drittel dieser Bildhälfte, eine fortfliegende Schwalbe (Abbildung 1). Auf Qi Baishis hochformatiger Skizze nimmt ein Wasserbüffel das untere Drittel des Fläche ein; hinter ihm erscheint in wenigen dünnen, langen Strichen die Andeutung eines Wasserfalls, die ganze Länge der Bildfläche hinabfallend, und in der Mitte des links davon freibleibenden Raumes ist ein Schwalbenpärchen in kunstvollem Flug zu sehen (Abbildung 2). Trotz des unterschiedlichen Formates fällt die übereinstimmende Entscheidung der Künstler ins Auge, dem massig-raumgreifenden und - bei aller Bewegtheit der Gesten - statischen eigentlichen Sujet als Kontrapunkt den ätherischen, Raum erzeugenden Flug der Schwalbe gegenüberzustellen.

Die Ähnlichkeit der Skizzen dieser beiden Künstler, deren Lebenszeiten aufeinander folgten, mag durchaus eher einer Homologie entspringen denn einer Analogie: Es ist nicht auszuschließen, dass Qi Baishi die Skizzen des eigentlich für seine Holzschnitte bekannten japanischen Künstlers kannte, und auf jeden Fall schließen beide an eine gemeinsame Malereitradition an, die selbstverständlich eine etablierte Formensprache hat, was die Möglichkeit der Formelhaftigkeit mit einschließt. Und dennoch - falls es eine solche Formel gäbe, was sich meiner Kenntnis entzieht, wäre sie erst recht ein Beleg für die Stimmigkeit meiner Annahme: dass die drückende Schwere der Hüte, mit denen die japanischen Unterhalterinnen ihre kunstvollen Frisuren abdecken, die Erdgebundenheit des Ochsen, der zum Himmel nur sehnsuchtsvoll hochsehen kann, die Gravitation abgeben, die die Schwalben an uns, die Betrachter, zurückbindet. Die Schwalbe ist kein Jenseitsvogel; ihr Flug überschreitet die Transzendenzschwelle nicht, aber er schreitet sie ab, um zurückzukehren. Die Parabel des Schwalbenflugs ist daher der Metapher wesensverwandt. Die Verbindung, die sich zwischen Li Bai und Chasper Po über das Motiv der Schwalbe auftut, ist - so die Grundthese dieses Essays - mehr als nur ein reiner Zufall; sie ist auch der Schwalbenhaftigkeit des Dichtens geschuldet.

Die genannte Verbindung besteht über zwei Gedichte von auffallend ähnlicher Motivik: Die Schwalben von Sent von Chasper Po und Der Abschied des Schwalbenpaars von Li Bai. Hier zunächst die beiden Gedichte: 


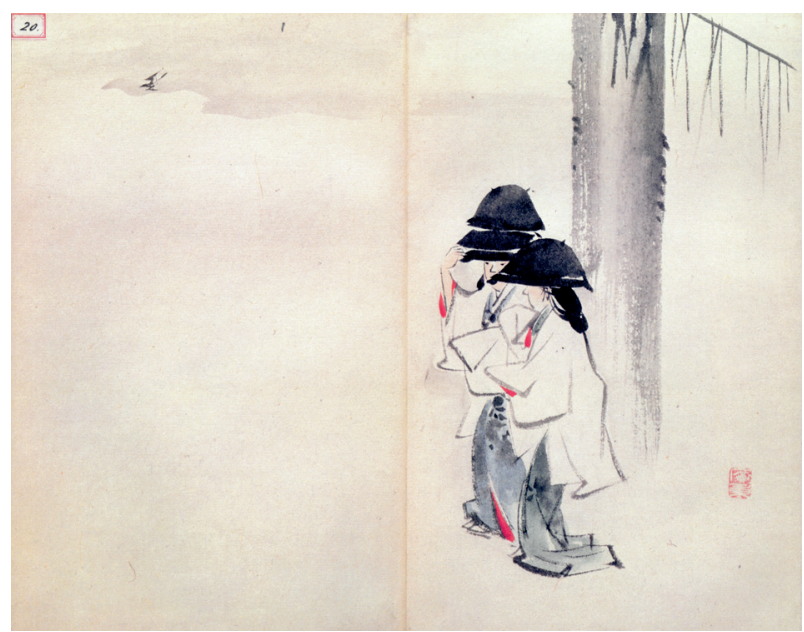

Abb. 1: Utagawa Hiroshige, o. T. (»Two Courtesans Promenading «), aus: The Sketchbooks of Hiroshige, New York: George Braziller, Inc., 1984., plate 20

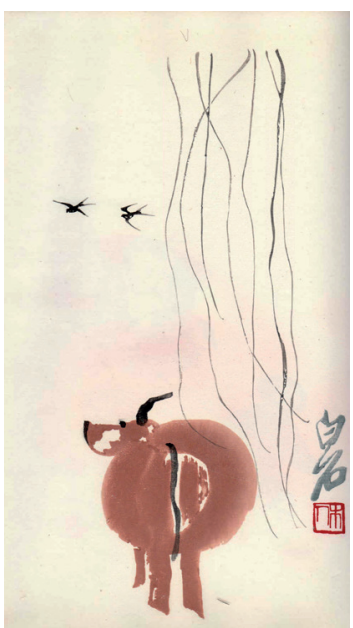

Abb. 2: Qi Baishi, o. T., aus: Beijing Rongbaozhai xinji shijian pu, Peking: Rongbaozhai, 1955, n. p.

\title{
Li Bai: Abschied der Schwalben
}

(Shuang yan li 雙燕離)

\author{
雙燕復雙燕，雙飛令人羙。 \\ 玉樓珠閣不獨棲，金窗繡戶長相見。 \\ 柏梁失火去，因入吳王宮。 \\ 吳宮又焚蕩, 㮲盡巢亦空。 \\ 憔悴一身在, 孀雌憶故雄。 \\ 雙飛難再得，傷我寸心中。2
}

Schwalbenpaar - und wieder das Schwalbenpaar, paarweise fliegen sie, wie beneidenswert.

Niemals einzeln hocken sie im schmucken Palast, stets sehen sie einander am goldenen Fenster.

Da brennt ein Feuer das Balkenwerk ab, sie ziehen weiter in den Palast des Königs von Wu.

Und wieder brennt der Palast die Küken tot, das Nest leer.

2 Li Bai: Li Taibo quanji [Vollständiges Werk von Li Bai], Taibei: He Luo tushu chubanshe, 1975, S. 112. 
Als einzige entkommen,

trauert das Muttertier um ihren Gemahl.

Nie wieder der paarweise Flug -

welche Pein für mein kleines Herz. ${ }^{3}$

\section{Chasper Po: La Rondolina da Sent}

La chattet bain la chà, la rondolina, mo seis vegl gniou nun ha'la plü chattà;

la chasa es ardütt'üna ruina,

be rest'ailch mür amo, s-chür, fümantà.

La rondolina svola disperada

mo ella voul il gniou rifabrichar

(il gniou prüvà ingio cha ell'es nada)

e seis fatschögn la glioud sta a guardar.

Seis svols guardand, sainta plü d'ün povret

plü greiv amo ch'eir el nun ha plü tet...

mo ella - vi' svoland - para sco dir:

»fains svelt! nu perdain temp! Reconstruir! « ${ }^{4}$

Die Schwalbe von Sent. Sie fand wohl das Haus, die Schwalbe, aber ihr altes Nest hat sie nicht mehr gefunden; das Haus ist zur Ruine heruntergebrannt, nur noch eine Art Mauer blieb übrig, dunkel, geräuchert. / Die Schwalbe fliegt verzweifelt, aber sie will das Nest wieder aufbauen (das warme Nest, wo sie geboren worden ist), und die Leute bleiben stehen und betrachten ihre Geschäftigkeit. / Ihre Flüge betrachtend, fühlt manch' ein Armer noch schwerer, dass auch er sein Dach verlor ... aber sie - im eiligen Flug - scheint zu sagen: »Machen wir vorwärts! Verlieren wir keine Zeit! Wiederaufbauen! ${ }^{5}$

Alle Übersetzungen aus dem Chinesischen in diesem Essay sind, soweit nicht anders angegeben, von der Verf.

Chasper Po: Rimas, hg. von Göri Klainguti und Clà Riatsch, Schlarigna: Uniun dals Grischs, 1996, S. 103.

Für die Interlinearübersetzung aus dem mir nicht geläufigen Rätoromanischen danke ich Mathias Gredig, dem überhaupt Dank gebührt dafür, mir das Thema `Schwalben` vorgeschlagen zu haben. Seinen Hinweisen auf eine Vielzahl weiterer rätoromanischer Gedichte mit Schwalbenthematik konnte ich leider an dieser Stelle nicht nachgehen. 
Das den Gedichten gemeinsame Motiv des abgebrannten Nestes fügt sich in unterschiedliche Narrative: In Li Bais dramatischerem Gedicht stehen die an Hiob erinnernde Dichte der Schicksalsschläge, vor allem aber die Zerstörung der Familienbande im Vordergrund; die Schwalben treten zunächst als Paar auf, und die tragische Schluss-Klimax des Gedichtes besteht im Verlust des Partners für das `Muttertier`. In Chasper Pos Gedicht erscheint die Schwalbe von Beginn an als eine einzelne, und im Mittelpunkt stehen die Beharrlichkeit, mit der sie mit den Folgen des Brandes kämpft, und der Lebensmut, den sie damit den Zuschauern mitteilt; die emotionale Kurve des Gedichts weist also nach oben.

Doch trotz der unterschiedlichen Erzählungen sind die Gemeinsamkeiten vielfältig, weit jenseits des verbindenden Grundmotivs. Auffallend ist schon die formale Ähnlichkeit: Die drei Strophen bei Chasper Po entsprechen exakt den drei Doppelversen bei Li Bai. Diese Gemeinsamkeit der Form resultiert in einer überraschenden strukturellen Analogie: in beiden Fällen steht diese Dreiheit für drei mouvements, drei parallel verlaufende Bewegungen. Beide Gedichte setzen ein mit einem auf den Schwalbenflug gerichteten Blick; der `Flug`(bei Chasper Po impliziert im `Suchen`) nimmt gleichermaßen Richtung auf eine Wohnstatt, auch wenn letztere bei Li Bai zunächst ein Palast mit goldenen Fenstern ist, bei Chasper Po von Anfang an eine rauchende Ruine. Die Mittelteile - Doppelvers 3 und 4 bzw. zweite Strophe - sind geprägt von Reiteration: Das Weiterziehen des Pärchens, das geschäftige Hin- und Herfliegen des Senter Schwälbchens beschwören die typische Hartnäckigkeit des animalischen Instinkts herauf, der das nicht zur Vollendung gekommene Geschäft fraglos wieder von vorn beginnt; bei Li Bai ad absurdum geführt durch die Reiteration auch des Schicksalsschlags. Im Schlussteil gehen in beiden Gedichten Schwalbe und lyrisches Ich eine enge emotionale Verbindung ein; hoffnungsfrohes Schaffen im einen Fall, tiefe Verzweiflung im anderen gehen direkt von der Schwalbe auf den Betrachter über. Sowohl in Li Bais als auch in Chasper Pos Schlusszeile verschmelzen die Stimme der Schwalbe und die des Dichter-Ichs, denn die zum Betrachter sprechende Schwalbe bei Chasper Po ist ja die Stimme des lyrischen Ich, das als Bewohner des abgebrannten Hauses das Geschick mit ihr teilt; und `mein Herz ` bei Li Bai lässt es offen, ob der Betrachter von sich in Identifikation mit der Schwalbe oder ob die Schwalbe selbst durch den Dichter spricht.

Wie kommt es zu diesen elementaren Verbindungen zwischen beiden Gedichten; kannte Chasper Po Li Bais Gedicht, antwortet er etwa darauf? Wahrscheinlich ist es nicht: La Rondolina da Sent ist auf ein Ereignis von 1921 datiert. ${ }^{6}$ Die älteste mir bekannte Übersetzung dieses Gedichts von Li Bai in eine westliche Sprache, von Franz Toussaint ins Französische, wurde 1920 publiziert; es folgten 1922 eine Übersetzung ins Englische von Shigeyoshi Obata und 1926 eine Übersetzung von Erwin von Zach ins Deutsche. ${ }^{7}$ Viel naheliegender als eine direkte Referenz ist also auch in diesem Fall eine wird eine Notiz des Autors zitiert, nach der Bezugspunkt des Gedichts der Brand des Hauses von Chasper Pult war. Chasper Pult (1869-1939), Romanist und Linguist, stammte genauso wie Chasper Po und dessen Freund und Dichterkollege Peider Lansel (1863-1943) aus Sent.

7 Erwin von Zach: Lit’aipos Gedichte. IV. Buch (37 lyrische Gedichte), in: Asia Major 3 (1926), S. 49-70, hier S. 52 f.; Li Bai: The Works of Li Po, the Chinese Poet, übers. von Shigeyoshi Obata, 
strukturelle Analogie. ${ }^{8} \mathrm{Zu}$ ihren Voraussetzungen gehört womöglich nicht nur das allgemeine Identifikationsangebot, das die familiäre Lebensweise der Schwalben für Menschen bereithält, sondern eine spezifische Nähe beider Dichter zu (diesen) Vögeln.

Chasper Po, der ein Gutteil seines Lebens in Italien verbrachte, gehörte damit zu einer Gruppe, die man heute als transnationale Migranten bezeichnen würde, die man aber im Engadin schlicht srandulins` ('Schwalben`) nannte. ${ }^{9}$ Dies allein legt bereits nahe, dass ein Gedicht über Schwalben aus seiner Feder kaum ohne eine Reflexion der eigenen 'Schwalbenhaftigkeit a auskäme; oder umgekehrt, dass die eigene Verbundenheit mit einer Heimat, die nicht selbstverständlich zur Verfügung steht, sondern immer von neuem (auf)gesucht werden muss, ihren Ausdruck im Bild der wiederkehrenden Schwalbe findet. Das Gedicht selbst fügt diesen Passungen aber noch eine weitere Ebene hinzu. Die Heimat, der die Schwalbe durch stete Wiederkehr die Treue hält, ist eine gefährdete und beschädigte, symbolisiert durch das abgebrannte Haus (eines von vielen in dem katastrophalen Brand von 1921). Wenn wir nun mit Heidegger als das eigentliche >Haus des Seins` die Sprache auffassen, so ergibt sich sofort eine evidente Parallele zwischen der Schwalbe, die »ihr warmes Nest, in dem sie geboren wurde«, unbeirrbar wieder aufbaut, und dem Senter Freundeskreis um (Chasper) Po, Peider (Lansel) und (Chasper) Pult, die sich der Konsolidierung der rätoromanischen Sprache verschrieben hatten. >Reconstruir kann dabei insbesondere für Chasper Pos eigenen, weniger konservativen als vielmehr konstruktiven Zugriff auf die Heimatsprache stehen. ${ }^{10}$

Auch in Li Bais Gedicht sehen Interpreten Bezüge zu seiner eigenen Lebenssituation. Der Übersetzer Obata nennt es »[a]nother allegorical poem«, und lässt uns wissen: »A commentator says that this is a fable of Li Po's own life, he with his hopes

New York: E. P. Dutton and Co., 1928 ['1922], S. 102. Die Übersetzung von Franz Toussaint in La Flute de Jade, Paris 1920, finde ich bei Obata angegeben (ebd., S. 229); sie liegt mir nicht vor. Laut Obata handelt es sich bei den Übersetzungen von Toussaint um »very free and often fragmentary translations in prose (ebd., S. 217), sodass vermutet werden kann, dass die Strukturen des Originals in dieser ältesten Version wenig durchscheinen. - In Obatas umfangreicher Liste früherer Übersetzungen von Gedichten Li Bais in westliche Sprachen fehlt Zach; möglicherweise gibt es also noch Übersehenes.

Beispiele solcher literarischer Analogstrukturen, die daraufberuhen, dass ein literarisches Motiv oder Motivbündel unter bestimmten Voraussetzungen eine bestimmte innere Logik entfaltet, sind auch anderweitig belegt. Ich selbst habe z. B. eine solche strukturelle Analogie zwischen Heinrich von Kleists (1777-1811) Käthchen von Heilbronn und Tang Xianzus (15501616) Mudanting (»Der Päonienpavillon «) beschrieben, vgl. Marion Eggert: Die Botschaften der Träume, in: Studien zum Traum der Roten Kammer (Hongloumeng), hg. von Wolfgang Kubin, Bonn: Bouvier, 1999, S. 41-59.

9 Clà Riatsch: Der Komiker und die Puristen. Mehrsprachigkeit und Sprachzensur im Werk von Chasper Po (1856-1936), in: Versants 27 (1995), S. 165-183, hier S. 166.

10 Vgl. ebd. sowie das Kapitel »Ein mahnender Dichter und ein spöttischer Reimer: Peider Lansel und Chasper Po" in Clà Riatsch: Pathos und Parodie. Inversionslagen in der bündnerromanischen Poesie, Aachen: Shaker Verlag, 2015, S. 81-96. Allerdings steht beim `reconstruir natürlich die pragmatische Seite im Vordergrund: Von 45 dem großen Brand von Sent zum Opfer gefallenen Häusern wurden $29 \mathrm{im}$ >Heimatbaustil« wiedererrichtet, also tatsächlich `rekonstruiert‘. Vgl. die Darstellung des Ereignisses auf der Webseite von Sent (www.sent-online.ch/ istorgia/incendis/incendi1921/incendi_1921.html, Zugriff 20.04.2020). 
and ambitions being compared with the mother swallow with her mate and younglings. $«^{11}$ Die genaueren Erläuterungen, die Obata bzw. der von ihm anonym zitierte Kommentar dafür anbringen, stützen diese Interpretation sicher, brauchen uns hier aber nicht zu interessieren; Gedichte als allegorische Selbstaussagen sind zu ubiquitär in der chinesischen (bzw. sinitischen ${ }^{12}$ ) Literatur, als dass entsprechende Lesungen eines eingehenden Beweises bedürften, um plausibel zu sein. Bedeutsamer für ein Aufspüren der inneren Parallele zwischen Li Bais und Chasper Pos Schwalbendichtung ist der Umstand, dass Vögel, wenn auch nicht unbedingt Schwalben, auch bei Li Bai eine über die Gedichtsituation hinausgehende Identifikationsfigur sind. Nicht nur gehört zur Charakterisierung Li Bais in der traditionellen chinesischen Biografik das Hochfliegende: "Von klein auf besaß er ein Talent zum Entgrenzten (yicai), und sein Sinn war ins Große und Weite gerichtet, sein Geist schwebte weit über der Welt" (so beschreibt ihn sein Biograf in der Alten Geschichte der TangZeit). ${ }^{13}$ Vögel jeder Art sind ein überaus häufiges Motiv seiner Dichtung, und Li Bai selbst rückt sich in einem autobiografischen Brief in große Nähe zur Vogelwelt:

\begin{abstract}
Einst zog ich mich gemeinsam mit dem Privatier (yiren) Dongyanzi in den Süden des Min-Gebirges zurück. Ich wohnte dort [wie] in einem Nest (chao ju) und betrat mehrere Jahre lang keine Stadt. Dort nährte ich tausende seltener Vögel; wenn ich sie rief, fraßen sie mir das Futter aus den Händen, ohne die leiseste Nervosität. ${ }^{14}$
\end{abstract}

Während der Dichter selbst in dieser Schilderung wie ausgewildert erscheint - seine Heimstatt ein Nest, kein Haus -, verbringt er seine Zeit damit, `seltene` Vögel, also Boten der Wildnis, zu domestizieren; ihre Zutraulichkeit ist zugleich Zeichen seiner Naturnähe. Die Grenze zwischen dem Wilden und dem Kultivierten, dem Organisch-Individuellen und dem gesellschaftlich Vermittelten ist zwischen dem vogelfreien Dichter und den dichterliebenden Vögeln nicht suspendiert, sondern stausend ‘-fach überschritten. Dieses Vermittelnde aber zwischen dem Heimischen, Bekannten, Domestizierten, und dem Fremden und Überraschenden ist, wie wir sahen, ein wesentlicher Zug genau dessen, womit die Schwalbe ihren menschlichen Betrachter beeindruckt.

Damit soll natürlich nicht gesagt sein, dass Li Bai sich in besonderer Weise jenseits des hier zur Diskussion stehenden Gedichts - gerade mit Schwalben identifiziert hätte. Ganz im Gegenteil: eher ist ihm das Bild des Phönix angemessen. Als solcher wird er im 762 geschriebenen Vorwort zur frühesten Sammlung seiner Gedichte charakterisiert: "All die Weisen zeigten ihm ihre Verehrung, so wie die [ge-

11 Li Bai: The Works of Li Po, S. 102.

12 Mit `sinitisch ist der chinesisch inspirierte Kulturraum (insbes. Vietnam, Korea, Japan) gemeint, in dem gerade die chinesische Dichtungstradition ungeheuer einflussreich war.

13 Liu Xu: Jiu Tang shu j. 190 xia.

14 »Shang Anzhou Pei zhangshi shu« [»Brief an Administrator Pei des Distrikts Anzhou«], in: Li Bai: Li Taibo quanji, S. 607. 
wöhnlichen] Vögel sich dem Phönix zuwenden.» ${ }^{15}$ Und Li Bai selbst zögert nicht, sich mit dem Phönix zu vergleichen. In einem Gedicht, in dem er offensichtlich die eigene Erfolglosigkeit in der Welt beklagt, heißt es:

»Im Kaiserbaum nisten Schwalben und Spatzen, während der Phönix im Dorngestrüpp haust. «16

Und in einem weiteren Gedicht lässt er die `Purpurschwalbe - Name eines edlen Rosses der Tradition - ausdrücklich hinter sich, indem er das eigene Bild als das eines Pegasus, eines Himmels-Pferds, entwirft. ${ }^{17}$

Angesichts dieses generellen Unwillens Li Bais, sich mit den Schwalben gemein zu machen, erweist sich die Parallele zwischen Chasper Pos und Li Bais Schwalbengedichten umso mehr als eine strukturelle. Auch wenn die Schwalbe dem Höhenflug des chinesischen Dichtergenies nicht entsprechen kann, kommt er doch nicht umhin, in den Zeichen ihres Fluges, oder in ihrem Flug als Zeichen, etwas Eigenes wiederzuerkennen. Vielleicht ist es das Erkennen dieser zeichnenden Bewegung, die den Rand des Bekannten abtastet, um dann doch ins Haus des Seins zurückzukehren, als der Bewegung des Dichtens selbst, das die Berührung zwischen dem himmelstürmenden Dichter der chinesischen Tang-Zeit und dem sich selbstironisch als simplen $>$ Reimer bezeichnenden rätoromanischen Dichter des 20. Jahrhunderts ermöglicht. Das geteilte Schwalbenmotiv stünde dann für das ebenso - bei allen Unterschieden in kulturell-historischer Umgebung und individueller Prägung - geteilte Potenzial der Dichtung, das Gebundene und das Ungebundene, das Zeichenhaft-Konventionelle und das Zauberhaft-Existenzielle der Sprache zu vereinen.

In diesem Zusammenhang sei, zu guter Letzt, auf eine weitere Gemeinsamkeit zwischen Li und Chasper hingewiesen: nicht das geteilte >Po < des Namens selbst, das sich einer inzwischen veralteten Umschrift des Chinesischen verdankte, ${ }^{18}$ jedoch der Umgang mit diesem Teil des Namens als Wort der Dichtung. In Li Bais Fall ist Bai oder eben $>$ Po $<$ der Eigenname. Er hat die Bedeutung `weiß und wurde ihm laut seiner Bio-

»Caotangji xu« [»Vorwort zur Strohhallen-Sammlung«], in: ebd., S. 706. Autor des Vorworts und Kompilator der Gedichtsammlung war Li Yangbing.

»Gufeng« [»Lieder im alten Stil«] Nr. 39, in: ebd., S. 67. Für eine Interpretation des Gedichts vgl. Paula M. Varsano: Tracking the Banished Immortal. The Poetry of Li Bo and its Critical Reception, Honolulu: University of Hawai'i Press, 2003, S. 177-179.

17 »Tian ma ge« (»Lied vom Himmelspferd«), in: Li Bai: Li Taibo quanji, S. 92. Auch der Pegasus bildet übrigens, ironisch gebrochen, eine Brücke zu Chasper Po. Auf dem Buchrücken der 1996er Edition seiner Gedichte ist folgendes Epigramm zu lesen:»Al Pegasus nun’s po dir `Hü / Be cur ch'el voul, l'armaint sta sü« (»Dem Pegasus kann man nicht `Hü« sagen / nur wenn er möchte, steht das Tier/Rind auf«).

18 Die in älterer westlicher Literatur in der Regel anzutreffende Schreibung $>$ Po $<$ folgte einer vormodernen Lautung des Zeichens sowie der Wade-Giles-Umschrift; in der von der Volksrepublik China geschaffenen und erfolgreich propagierten pinyin-Umschrift wäre >Bo< die korrekte Schreibung dieser (heute veralteten) Lesung des Schriftzeichens. Die moderne Standard-Aussprache des entsprechenden Zeichens ist `baiı, sodass der Dichter heute zumeist als Li Bai geschrieben wird. Dieser Essay bezog aus der im Call for Papers verwendete Schreibung Li Po für den Dichter Inspiration, nutzt aber die pinyin-Umschrift des Chinesischen. 
grafen verliehen, weil die Mutter in der Nacht der Geburt vom `Großen Weiß`, der Venus (Abend- bzw. Morgenstern), geträumt habe. ${ }^{19}$ Ohne den Bezug zu Lis Eigennamen herzustellen, macht nun der namhafte chinesische Literaturwissenschaftler Yuan Xingpei $\left({ }^{*} 1936\right)$ darauf aufmerksam, dass Li das Wort >weiß aus häufig verwendet. »Wohl weil er die Reinheit so liebt«, könne »unter seinem kreativen Pinsel so gut wie alles weiß werden «. ${ }^{20}$ Tatsächlich aber dürfte es sich in manchen der Fälle um eine Art augenzwinkernden Selbstbezug handeln - so etwa in dem bereits herangezogenen »Lied vom Himmelspferd «, in dem es heißt:

Wenn das Himmelspferd wiehert, so fliegen die Drachen davon; in seinen Augen leuchtet die Venus, seine Schultern gleichen Flügeln. [...] Mit seiner entgrenzten Energie (yiqi) durchmisst es die Neun Gefilde. Weiße Jade aufgehäuft zu Bergen: wer wagte, sie abzugraben? ${ }^{21}$

Die Venus (changgeng) - wie wir hörten, Li Bais Geburtsstern - wird auch >Großes Weiß genannt, taibai. Taibai ist aus diesem Grunde auch Li Bais Mannesname. Wenn das Himmelspferd, in dessen Augen man taibai/Taibai strahlen sieht, seine >entgrenzte ${ }^{22}$ Energie spielen lässt, türmt sich >weiße Jade` auf, über die kein anderer zu verfügen vermag. Was sollte diese `weiße Jade` anderes sein als Pos poetische Juwelen? Nicht anders, wenn auch leichter nachvollziehbar, macht Chasper Po seinen Nachnamen zum Wort in einem kurzen Reim, mit dem wir ihm das letzte Wort lassen wollen, auch wenn es sich um einen Prolog handelt:

Sch'eir na güsta »comme il faut «, Cuort faràl - plü cuort ch'el po!

Wenn auch nicht eben »comme il faut", kurz wird er es halten - so kurz, wie er es eben kann/po! ${ }^{23}$

19 Derartige Schwangerschafts- oder Geburtsträume sind in sinitischen Kulturen ein üblicher Bestandteil nicht nur der Hagiografien berühmter Persönlichkeiten, sondern auch des tatsächlichen Traumverhaltens bis mindestens ins 20. Jh. Vgl. Frederic James Seligson: Traditional Korean Birth Dreams: in: Korea Journal 28/1 (1988), S. 28-38.

20 Yuan Xingpei: Zhongguo shige yishu yanjiu [Zur chinesischen Dichtkunst], Peking: Beijing daxue chubanshe, 1987. Leider konnte ich das Buch nicht einsehen; ich stütze mich auf einen Auszug im Internet: http://old.pep.com.cn/gzyw/jszx/tbjxzy/kbjc/jsys/zggdsgswxs/201102/ t20110217_1021570.htm (Zugriff 08.04.2020). Der Auszug stammt aus dem Kapitel »Li Bais Dichtung und die Kultur der Hohen Tang-Zeit«.

21 Li Bai: Li Taibo quanji, S. 92.

22 Dieses rentgrenzt $<(y i)$ ist das gleiche Wort, das in seiner Biografie sein Talent (yicai) und in seinem autobiografischen Brief sein Alter Ego, den `Privatier` (yiren), bezeichnet.

23 Clà Riatsch: Minchatant, per üna jada / nu fa mal üna `pizchada‘. Our dad ün relasch da Chasper Po (1856-1936), in: Annalas da la Societad Retorumantscha 130 (2017), S. 109-130, hier S. 113. Wiederum danke ich für die Übersetzung Mathias Gredig. 


\section{Literatur}

Eberhard, Wolfram: Lexikon chinesischer Symbole. Geheime Sinnbilder in Kunst und Literatur, Leben und Denken der Chinesen, Köln: Diederichs, 1983.

Eggert, Marion: Die Botschaften der Träume, in: Studien zum Traum der Roten Kammer (Hongloumeng), hg. von Wolfgang Kubin, Bonn: Bouvier, 1999, S. 41-59.

Li Bai: The Works of Li Po, the Chinese Poet, übers. von Shigeyoshi Obata, New York: E. P. Dutton and Co., 1928 ['1922].

Li Bai: Li Taibo quanji [Vollständiges Werk von Li Bai], Taibei: He Luo tushu chubanshe, 1975.

Liu Xu: Jiu Tang shu [Alte Geschichte der Tang-Zeit], https://zh.wikisource.org/wiki/\%E8\% 88\%8A\%E5\%94\%90\%E6\%9B\%B8/\%E5\%8D\%B7190\%E4\%B8\%8B (Zugriff 08.04.2020).

Po, Chasper: Rimas, hg. von Göri Klainguti und Clà Riatsch, Schlarigna: Uniun dals Grischs, 1996.

Qi Baishi/Zhenduo Zheng: Beijing Rongbaozhai xinji shijian pu, Peking: Rongbaozhai, 1955.

Riatsch, Clà: Der Komiker und die Puristen. Mehrsprachigkeit und Sprachzensur im Werk von Chasper Po (1856-1936), in: Versants 27 (1995), S. 165-183.

Riatsch, Clà: Pathos und Parodie. Inversionslagen in der bündnerromanischen Poesie, Aachen: Shaker Verlag, 2015.

Riatsch, Clà: Minchatant, per üna jada / nu fa mal üna ‘pizchadaく. Our dad ün relasch da Chasper Po (1856-1936), in: Annalas da la Societad Retorumantscha 130 (2017), S. 109130

Seligson, Frederic James: Traditional Korean Birth Dreams: in: Korea Journal 28/1 (1988), S. 28-38.

Sent-Online: Incendi dals 8 gün 1921 / Dorfbrand vom 8. Juni 1921, www.sent-online.ch/istor gia/incendis/incendi1921/incendi_1921.html (Zugriff 20.04.2020).

Utagawa Hiroshige: The Sketchbooks of Hiroshige, New York: George Braziller, Inc., 1984.

Varsano, Paula M.: Tracking the Banished Immortal. The Poetry of Li Bo and its Critical Reception, Honolulu: University of Hawai'i Press, 2003, https://doi.org/10.1515/9780824865276.

Yuan, Xingpei: Zhongguo shige yishu yanjiu [Zur chinesischen Dichtkunst], Peking: Beijing daxue chubanshe, 1987.

Zach, Erwin von: Lit’aipos Gedichte. IV. Buch (37 lyrische Gedichte), in: Asia Major 3 (1926), S. 49-70.

Marion Eggert studierte Sinologie, Ethnologie und Japanologie in Heidelberg, München, Nanjing und Seoul, promovierte (1992) und habilitierte (1998) in München und absolvierte 1994/95 ein post-doctoral fellow am Korea Institute der Harvard University. Seit 1999 ist sie Professorin für Koreanistik an der Ruhr-Universität Bochum. Neben Übersetzungen koreanischer und chinesischer Literatur ins Deutsche und Englische publizierte sie zu einer Vielzahl von Themen der Literatur- und Geistesgeschichte, insbesondere zu Reiseliteratur, Traum und Subjektivität sowie epistemischen Konstellationen. 


\title{
Der doppelte Po und die Musik
}

Rätoromanisch-chinesische Studien, besonders zu

Li Po, Harry Partch und Chasper Po

\author{
Herausgegeben von \\ Mathias Gredig, Marc Winter, \\ Rico Valär und Roman Brotbeck \\ Redaktionelle Mitarbeit \\ Daniel Allenbach
}

Königshausen \& Neumann 
Bibliografische Information der Deutschen Nationalbibliothek

Die Deutsche Nationalbibliothek verzeichnet diese Publikation in der Deutschen

Nationalbibliografie; detaillierte bibliografische Daten sind im Internet über http://dnb.d-nb.de abrufbar.

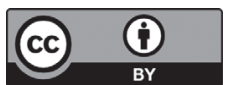

Dieses Werk ist lizenziert unter der Creative Commons Attribution 4.0 Lizenz (BY). Diese Lizenz erlaubt unter Voraussetzung der Namensnennung des Urhebers die Bearbeitung, Vervielfältigung und Verbreitung des Materials in jedem Format oder Medium für beliebige Zwecke, auch kommerziell. (Lizenztext: https://creativecommons.org/ licenses/by/4.0/deed.de) Die Bedingungen der Creative-Commons-Lizenz gelten nur für Originalmaterial. Die Wiederverwendung von Material aus anderen Quellen (gekennzeichnet mit Quellenangabe) wie z. B. Schaubilder, Abbildungen, Fotos und Textauszüge erfordert ggf. weitere Nutzungsgenehmigungen durch den jeweiligen Rechteinhaber.

Erschienen 2021 im Verlag Königshausen \& Neumann GmbH

(C) bei den Autoren

Die Druckvorstufe dieser Publikation wurde vom Schweizerischen Nationalfonds zur Förderung der wissenschaftlichen Forschung unterstützt.

\section{FNSNF}

SCHWEIZERISCHER NATIONALFONDS

ZUR FÖRDERUNG DER WISSENSCHAFTLICHEN FORSCHUNG

Wir danken der Kulturförderung des Kantons Graubünden.

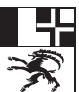

Kulturförderung Graubünden. Amt für Kultur

Promoziun da la cultura dal Grischun. Uffizi da cultura

Promozione della cultura dei Grigioni. Ufficio della cultura

SWIISSLOS

Hochschule der Künste Bern

www.hkb.bfh.ch

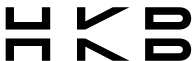

Hochschule der Künste Bern

Haute école des arts de Bern

Bern University of the Arts

Umschlag: skh-softics / coverart

Umschlagabbildung: Lea Gredig

Print-ISBN 978-3-8260-7180-5

PDF-ISBN 978-3-8260-7233-8

DOI $10.26045 /$ po

https.//doi.org/10.36202/9783826072338

Gedruckt auf säurefreiem, alterungsbeständigem Papier

Printed in Germany

www.koenigshausen-neumann.de

www.ebook.de

www.buchhandel.de

www.buchkatalog.de 


\section{Inhalt}

Prolog

Dumenic Andry

Chasper Pos Humor

Renzo Caduff

Chasper Pos rhythmische Versgestaltung - eine shinkende Mähre`?

Rico Valär

Rätoromanische Nachdichtungen chinesischer Lyrik bei

Gian Fadri Caderas und Peider Lansel

Eine Spurensuche

Mathias Gredig

China in rätoromanischen Zeitungen, Zeitschriften

und literarischen Texten

Marion Eggert

Schwalbenflug in Gedichten von Li Bai und Chasper Po

Thomas Geissmann

Die Rolle der Gibbons beim chinesischen Dichter Li Bai

Marc Winter

„Chinas Dichterfürst«

Die Rezeption Li Bais als literarischer Superstar im Westen

Eva Schestag

"A most difficult man«

Ezra Pound als Übersetzer von Li Bai, mit einem Seitenblick auf Shigeyoshi Obata

Odila Schröder

Chinesische Li-Bai-Vertonungen in Jahren der Unruhe 
Mathias Gredig

Quantitative Überlegungen zum Phänomen

der Li-Bai-Vertonungen im Westen

Mit Beobachtungen zu drei Vertonungen des Gedichtes

Chun ye Luo cheng wen di (In einer Frühlingsnacht in Luoyang eine Flöte hören)

\section{Gesine Schröder}

"Die Hüften schwingen sich nun nicht mehr»

Li-Bai-Vertonungen von Komponistinnen

Heinrich Aerni

Li-Bai-Vertonungen in der Schweiz

Matthias Schmidt

Übersetzung ohne Original?

Gustav Mahler, Anton Webern und Li Bai

\section{Christoph Haffter}

Szenen der Selbstenttäuschung

Hanns Eislers Die rote und die weiße Rose nach Li Bai und die Antinomien der Kriegslyrik

Thomas Meyer

„Wunderlich im Spiegelbilde»

Zu einigen Vertonungen des Pavillon-Gedichts

\section{Mathias Gredig}

Gedanken über Li Bais Jing ye si (Gedanken in einer stillen Nacht)

und dessen Vertonungen im Westen

\section{Martin Skamletz}

"I've turned into a great reviser."

Lee Hoibys Vertonung von Li Bais The River-Merchant's Wife: A Letter

und ihr Bezug zu Harry Partch

\section{Martin Skamletz}

"Of course I am a weak shadow of Lee Hoiby as a Kitharist."

Five letters by Harry Partch, 1948-1958

\section{Marc Kilchenmann}

Ben Johnstons Verhältnis zu Harry Partch und seine Three Chinese Lyrics 
Eleni Ralli

Parallelen und Modifikationen der Notation in verschiedenen Quellen

von Harry Partchs Seventeen Lyrics by Li Po

Schwierigkeiten und Transkriptionsvorschläge

Charles Corey

Gesture and Intention in the Art Songs of Harry Partch

Caspar Johannes Walter

Sprechmelodie als Quelle von Melodik und Harmonik

The Intruder aus Harry Partchs Li-Bai-Vertonungen

Roman Brotbeck

Der Sprechgesang bei Arnold Schönberg und Harry Partch

Eine Annäherung

Namensregister 